\section{THE MANAGEMENT OF THE PNEUMONIAS IN ADULTS}

\author{
By James Maxwell, M.D., F.R.C.P.
}

(Asst. Phys. St. Bartholomew's Hospital; Phys. Royal Chest Hospital etc.)

The treatment of acute respiratory infections has altered in many respects during the last decade, and it is more than probable that the immediate future will show still further changes, although it is likely that these will be more in the nature of refinements than alterations in general principles.

Not many years ago an article on the treatment of the pneumonias would have laid stress chiefly on the nursing and general management of the patient and would have dwelt but little on the use of drugs. At the present day we have drugs available which will control the fever and cut short the duration of the illness in the majority of the cases, but the nursing and general management of the patient still maintain much of their old importance and must still be given first mention in any discussion on treatment.

It is a maxim that any patient with an acute respiratory infection associated with rise of temperature must be nursed in bed and must remain at rest until the temperature has been normal for a sufficient period of time. The common mistake is to allow the patient to get up too soon, and the penalty is often a relapse. Even with a very short duration of fever the patient should remain in bed for at least 48 hours after the temperature has fallen to normal and, if there has been evidence of consolidation in the lung, this period should be proportionately increased; a temperature which has lasted for a week should be followed by rest in bed for a further week. In this respect a very useful guide is the sedimentation rate, which is always considerably increased with an acute infection in the respiratory tract. Repeated observation at intervals of two or three days will show a progressive fall in the rate as the condition of the patient improves; the test is delicate and it yields information which is very helpful to the clinician, in that it may remain raised for some considerable period after the temperature has fallen, and when the patient may protest that he feels well. Experience shows that it is unsafe to allow an apparently convalescent patient to be up and about while the sedimentation rate is raised appreciably above the normal upper limit.

General nursing care is apt to be taken for granted, but it should always be supervised by the physician in charge and the details must be carefully carried out. The bedroom should be well aired, but not draughty, and should be maintained at an even temperature of about $60^{\circ} \mathrm{F}$. It is desirable that the patient should take plenty of liquids, but the present view is that fever by itself is not a contra-indication to giving solid food. The patient who is not acutely ill and whose appetite is not impaired may be allowed a simple light diet. In this connection it may be mentioned that any considerable quantity of raw milk is not easily digested by the febrile patient, for it is very likely to cause a considerable degree of flatulence and abdominal discomfort, and these are to be avoided at all costs in dealing with the more serious acute respiratory infections. It is much better therefore that the fluids should consist of fruit juices, tea and other bland liquids, while sugar may be given in considerable quantity with benefit.

The management of the bowels is a matter which requires careful supervision and considerable experience in deciding when to act and when to leave well alone. So long as the abdomen is not distended and there is no discomfort it is better not to interfere in any way. Flatulence and distension are indications that action must be taken, and a simple enema is far less disturbing to the patient than purgation by means of drugs. A moderate degree of distension can often be relieved by the application of a turpentine stupe.

There are certain other indications for routine treatment in pneumonia. Pain in the chest, from acute pleurisy, is very often present and may inter fere with sleep. This is best treated by the locas application of heat in the form of anti-phlogistine or, better still, by the old-fashioned linseed meal poultice; the advantage of the latter is that it is much cleaner and retains its heat for a considerably longer period. In more severe cases counterirritation may be applied by cupping, by blistering, or by the local application of leeches. Insomnia is sometimes a troublesome symptom which may need treatment in order to prevent the onset of physical exhaustion. It is a good rule to ensure sound sleep in acute respiratory disease, and for this purpose there is no drug which is more certain in its effect than opium. Most of these patients tolerate morphia well and, in spite of a certain natural diffidence in the prescription of a very potent drug to an ill patient, the advantages far more than outweigh any possible objection to its use. It is rarely necessary to give morphia for more than three or four consecutive nights, so that there is no risk of habit formation. If the general condition of the patient in any way contra-indicates the use of morphia, a safe and efficient drug for promoting sleep is paraldehyde, which can be given in doses of 2 drachms by the mouth. In case of necessity it may also be given per rectum or intramuscularly. The patient who is accustomed to take alcohol regularly should usually be given a limited quantity throughout the acute stages of the 
illness. It is often found that such patients will sleep quite well after a moderate dose of spirits. Heavy drinkers are liable to develop delirium tremens during an attack of pneumonia and alcohol must always be supplied early in such cases.

The routine prescription of a cough mixture is hardly necessary in the average case. A cough which is out of proportion to the quantity of sputum brought up, which produces a raw feeling under the sternum, and which disturbs the patient, may well be relieved by a simple alkaline mixture.

$\mathrm{R}$

$\begin{array}{lll}\text { Sod. bic. ... } & \ldots & \text { Gr.Io } \\ \text { Tr. ipecac. .. } & \ldots & m_{5} \\ \text { Tr. opii. camph. } & \ldots & m_{\text {Io }} \\ \text { Syr. tolu. . } & \ldots & m_{30} \\ \text { Aq. anisi. ad } & \text {.. } & \text { oz. } \frac{1}{2}\end{array}$

four hourly.

In the milder cases an occasional dose of a simple linctus, such as Gee's Linctus, may be prescribed. Cough mixtures which contain the more irritant drugs, such as salts of ammonia, squills and senega should be avoided, as their chief action is irritant and the most that they are likely to accomplish is to upset the digestion. The administration of oxygen used to be regarded as part of the routine treatment of pneumonia, and few patients escaped the discomfort of a nasal tube. It is quite clear that there is only one indication for the use of oxygen in pneumonia, and that is the presence of cyanosis. Actually a slight degree of cyanosis in the early stages is not necessarily of serious significance, for it may only mean that a quantity of venous blood is still circulating through the solid lobe and has not been aerated before it reaches the arterial circulation; oxygen is unlikely to influence cyanosis which is due to the presence of such a "shunt." When there is reason to believe, however, that cyanosis is due to inefficient aeration or to heart failure or, as is more common, to a combination of both these factors, the administration of oxygen becomes imperative. The modern method is to supply oxygen in high concentration through a B.L.B. mask, which many patients tolerate quite well. It is simple to use and any required concentration of oxygen may easily be obtained by suitable adjustment of the regulating mechanism. Very occasionally a patient may be unable to tolerate the mask and in such cases it is perhaps best to rely on the nasal catheter, which is not nearly so efficient but which nevertheless has given good service for many years. The oxygen tent was widely used some years ago but it is a cumbersome piece of mechanism which requires expert handling and constant supervision; it is likely that this method will fall into disuse in view of the recent developments of simpler and more efficient apparatus.
Perhaps the oldest routine treatment of pne monia was the operation of venesection. Bloo letting is now very rarely practised and yet it has its place in treatment. Cyanosis, pulmonary congestion, and evidence of commencing right he⿳亠्冖⿰t⿱⺊口灬t failure are the special indications, and the timety removal of a pint of blood may make a consideralge difference to the ultimate outcome of the attack. The blood is often rather viscid and a venesecti夰 may prove difficult if carried out by the ordina? means. It is better, if there should be the slightest difficulty, to allow the blood to flow from an $\frac{\mathrm{m}}{\mathrm{B}}$ cision in the vein.

Until fifteen years ago the treatment of pnesmonia had altered but little for a century or mote. The first great advance was the introduction of specific serum which produced an immediate improvement in the results of treatment, but $\rightarrow \mathrm{t}$ speedily became apparent that there were seveed 1 quite serious disadvantages. In the first place the serum was type-specific, so that sputum had to $\overrightarrow{b e}$ obtained and the type of pneumococcus detemined before the serum could be employed. Various methods were devised by which this formation could be obtained in a reliable manner a short space of time, yet even the simplest methoys demanded apparatus and experience which were not within the reach of most practitioners. of next difficulty was that severe serum reactions common, and sudden death was not unknown. Th fact it was sometimes doubtful whether the ilfness caused by the serum was less severe than the which would have resulted if the pneumonia hagd been left to run its course. The final difficu傮y was the expense of the treatment, which was very considerable. Nevertheless serum treatment wougd have flourished unless it had been superseded bysa method which was intrinsically simpler and mege efficient.

The discovery of the action of drugs of the sulphonamide series on the pneumococcus opened a new and much more promising field. The original compound to be used on an extensive scife was sulphapyridine, and this proved immediatey effective in a great many cases, but it is to be notod that the results of chemotherapy are much mo striking in the younger age groups. With sulplapyridine it was possible to control the temperaturite within 48 . hours and the course of the illness waxs often cut short in a most dramatic manner. not the same time it became apparent that there wabe disadvantages attached to the use of this drug Digestive disturbances were common, skin rashies were seen in a number of cases, and the appearariee of cyanosis caused anxiety at first because it whe thought that one action of the drug was to producice a state of sulphaemoglobinaemia; it is now knopgn that this fear was ill-founded, and that nothiag 
worse than methaemoglobinaemia results from the use of sulphapyridine. Various drugs, such as methylene blue, were given in order to diminish the cyanosis, and nicotinic acid to prevent the digestive disturbances, and varying degrees of success were reported. Meanwhile the search for new compounds continued and others have been evolved, of which sulphadiazine and sulphamezathine are those now in most common use. As sulphamezathine is produced in this country and as it is relatively cheaper than many of the other compounds, it is likely to be employed on an increasing scale until, in its turn, it is supplanted by some new compound, even more efficient and less toxic. One great advantage of sulphamezathine is that it is less insoluble than some of the other sulphonamides, so that the risk of kidney damage is considerably lessened when it is used. At the same time it is a little less powerful than sulphapyridine and the initial dose should be rather higher, 3 or 4 grms., by the mouth. This should be followed by 2 grms. six-hourly until, the temperature has been normal for 48 hours. A full course should therefore consist of between 30 and 40 grms. of the drug, given over a period of four or five days. In very acute cases it may be necessary to administer sulphamezathine either intravenously or intramuscularly, in which case the intial dose is I grm., the subsequent doses being given by the mouth as stated above. It is unnecessary to consider the use of the other members of this series or the adoption of special methods to overcome the side actions of sulphamezathine as these are practically non-existent, although rashes and "drug-fever" are thought to occur. In connection with the use of drugs of the sulphonamide series a word of warning must be given that, in order to obtain the desired effect, it is necessary to give the drug in full doses. There would appear to be little doubt that such complications as delayed resolution, chronic pleural effusion and sterile empyema are much more likely to occur when inadequate doses have been prescribed and when the pneumococcus has been allowed to become sulphonamide resistant.

The much more recent introduction of penicillin may again cause a radical alteration in the management of pneumonia, for the pneumococcus is one of the organisms which are very sensitive to this drug. There have so far been few opportunities for physicians in this country to acquire any great experience of the treatment of pneumonia by this substance, for the supply of penicillin has been greatly restricted and it has naturally been used more for surgical than for medical cases. Reports, however, have been published of the results in small series of cases, and a good deal of information has also been received from the United States. It would appear to be certain that penicillin is most effective in the treatment of pneumonia. In fact it is possible that this will become the routine treatment of the future, although its use involves injections, whereas the sulphonamides are given by the mouth. At least it can be stated that penicillin is effective in a number of cases in which the pneumococcus proves to be sulphonamide resistant, and therefore it will always have a place in the treatment of pneumonia, even although further improvements in chemotherapy show that this method is better for routine use.

It cannot be considered that the method of administration of penicillin is as yet established and, in fact, there are two main possibilities. In the first place the drug may be given by means of intramuscular injections and, as the substance is rapidly destroyed, these must be given at threehourly intervals during the day. The usual dose is ro,00o units and the treatment is carried on for 3 or 4 days, so that more than 150,000 units are necessary in order to overcome an average attack of pneumonia; it is quite possible that this dosage will be found to be below the optimum and that larger doses will be advised in the future. In more serious cases, especially when there is evidence of bacterial invasion of the blood stream, it is better to give the first two injections by the intravenous route, giving 25,000 units at three-hourlyo intervals. The drug can then be continued intra? muscularly as before.

Alternatively it is possible to give penicillin by? means of a continuous intramuscular drip. In this case the total quantity given during the day is much the same as that already indicated. The advantage of the continuous drip method is that a steady concentration of the drug can be obtained, but the apparatus is a little complicated and the patient must be kept under continuous and expert supervision. It is quite likely that this will prove in the future to be the method of choice, especially for patients who are being nursed in hospital, but it is not so certain that it is the best method for patients who are being looked after at home. The three-hourly injection is definitely less efficient, for the concentration varies considerably and the injections are often painful, so much so that some patients find them difficult to tolerate; nevertheless it is easy to carry out this method when conditions of treatment are not ideal and, for the $N$ present at least, it will prove to be the method of choice in many cases.

The great advantages of penicillin are that toxic $\mathbb{\omega}^{N}$ complications are almost unknown, although it has been suggested that urticaria is an occasional 0 sequel. Penicillin has been shown to be effective in cases in which the pneumococcus has proved to $\stackrel{?}{+}$ be sulphonamide resistant.

A point which applies both in the case of peni- 
cillin and sulphonamide is that the effects of treatment are much more beneficial in the younger age groups. In elderly patients the pneumonia is often superposed on some underlying chronic disease, and in this type of case the resistance is at a low $\mathrm{ebb}$, so that the powers of recuperation may not prove sufficient to enable the patient to withstand the infection. Care must therefore be exercised in framing a prognosis, and the history of the previous health in the immediate past should be taken into account.

Of recent years it has been recognised that the commonest acute inflammatory consolidation of the lung is not true pneumonia, but pneumonitis ("primary atypical pneumonia"). The clinical distinction is easy to make because the respiration rate in pneumonitis is little raised. It is very important that the correct diagnosis should be made and that a loose diagnosis of "pneumonia" should be avoided, for the prognosis in pneumonitis is excellent and very little treatment is needed. It is not yet certain whether the condition is always due to a virus infection or whether it may on occasion result from a pneumococcal infection of exceptionally low virulence, but it is possible that both types of cases may be at present included in the one group. The treatment is always general and is on the lines already laid down in the first part of this article. It is generally agreed that the sulphonamide group of drugs is not effective in pneumonitis and, in any case, the illness tends towards spontaneous recovery with few complications. Of the drugs recommended perhaps the only one which is likely to be beneficial is quinine, and this is always worth a trial. The action of penicillin in pneumonitis is as yet unknown, but it would hardly seem likely that so potent a drug will ever be really needed in a condition which runs so benign a course.

\section{THE DIAGNOSIS AND TREATMENT OF PNEUMONIA IN INFANCY AND CHILDHOOD}

\section{By W. G. Wyllie, M.D., F.R.C.P.} (Phys. Hospital Sick Children, Great Ormond Street)

First let it be clearly understood that pneumonia in early childhood is often a preventable disease. It is among the children of the poorer classes that the bulk of the case-incidence occurs. In any local hospital one comes to know too well the districts, the families, from which one or more sibs, infants or toddlers, are admitted for treatment, some with recurrent attacks. Social medi- cine, or the study of positive health, will have great part to play in aiming at better housing proper clothing and feeding, dispelling ignoranc $\mathbb{\mathscr { Q }}$ on food values, and providing an adequate vitamire intake. The natural resistance of the ill-fed under-nourished, under-vitaminised child is below? par, and though florid rickets is now seldom seenc its old associate the chronic catarrhal state of the upper respiratory passages is still too common $\frac{\bar{\Phi}}{\bar{D}}$ By loading bacterial infection on lowered resist $\mathbb{\square}$ ance, the catarrhal state is readily transformed into a pneumonia.

A further word on prevention: it may be denied that the prenatal diet of the mother affects the health of the infant unless extreme deficiencies are present. Yet Ebbs, Tisdall and Scott have given strong reasons to believe that by providing. expectant mothers, having a low income and on poor diet, with dietary supplements, the incidences of illness in their infants up to six months of age्ల can be reduced. In a control group without supple ments to the maternal diet infection was presen $\vec{b}$ in $3 \mathrm{I}$ per cent of the infants as against 8 per cent oP infants of mothers on supplemented diets. In the control group the types of illness in the infants up to six months of age in order of frequency werद्ध anaemia, frequent colds (catarrh), dystrophy? rickets, pneumonia, and bronchitis. The figste three or four of these prepare the ground for easy inoculation of the bacterial infections.

When it is stated that pneumonia has its highese mortality at the two extremes of life, the states ment as it affects infancy must be qualified. It is the debilitated infant or young child whose chances of recovery are reduced, as its pneumonia is $\mathbb{2}$ complication of ill-health and often of measles o $\overrightarrow{\vec{b}}$ of whooping cough. These are the cases with $\vec{\not}$ high mortality in which chemotherapy often dis? appoints. But with an uncomplicated pneumonia a previously healthy infant in my experience has as good a chance of recovery by chemotherapy as the older child or adult.

The traditional division of pneumonia int lobar and broncho-pneumonia as separate entitie is losing ground, and is even passing from the latest text-books-a sure sign of decay! It is better to speak of Primary and Secondary pneu $\rightarrow$ monia, in either of which the anatomical distri․ㅡㄹ bution may take the lobar or the bronchial formin their incidence varying at different ages. Broncho= pneumonia is especially a disease of early infancys even as a primary. pneumonia; afterwards mores and more primary cases take the lobar formo The primary pneumonia is caused by the inhalation of recoverable or unknown bacteria and viruses币 Most often the noxus is a member of the pneumo? cocci, less often a haemolytic streptococcus or ao haemophilus influenzae, rarely the staphylococcus 